\title{
Generalized Mean-Field Fractional BSDEs With Non-Lipschitz Coefficients
}

\author{
Qun Shi \\ Correspondence: School of Mathematics and Statistics, Jiangxi Normal University, Nanchang, Jiangxi, P.R.China
}

Received: February 4, 2021 Accepted: April 9, 2021 Online Published: April 13, 2021

doi:10.5539/ijsp.v10n3p77 URL: https://doi.org/10.5539/ijsp.v10n3p77

\begin{abstract}
In this paper we consider one dimensional generalized mean-field backward stochastic differential equations (BSDEs) driven by fractional Brownian motion, i.e., the generators of our mean-field FBSDEs depend not only on the solution but also on the law of the solution. We first give a totally new comparison theorem for such type of BSDEs under Lipschitz condition. Furthermore, we study the existence of the solution of such mean-field FBSDEs when the coefficients are only continuous and with a linear growth.
\end{abstract}

Keywords: backward stochastic differential equation, continuous coefficients, comparison theorem, fractional Brownian motion, mean-field

Mathematical subject classification: 60H05, 60H07.

\section{Introduction}

General backward stochastic differential equations driven by a Brownian motion were first studied by Pardoux and Peng (1992). Later Pardoux and Zhang (1998) introduced the generalized BSDEs,i.e. BSDEs with an additional term-an integral with respect to an increasing process. Backward stochastic differential equations driven by a fractional Brownian motion with $H \in(1 / 2,1)$ were first considered by Biagini,Hu,Øksendal and Sulem (2002), where they studied the stochastic maximal principle in the framework of a fractional Brownian motion. By adapting the four-step scheme introduced by Ma,Protter and Yong(1994) and the so-called S-transform, Bender (2005) stuided BSDEs driven by a fractional Brownian motion with $H \in(0,1)$. Indeed, throughout a backward parabolic PDE, he constructed an explicit solution of a kind of linear fractional BSDE. Hu and Peng (2009) were the first to study nonlinear BSDEs governed by a fractional Brownian motion.

It is well known that backward stochastic differential equation provided stochastic representation of solution of some classes of partial differential equations of second order. With the help of backward stochastic differential equations with respect to a Brownian motion and a Poisson random measure, some authors generalized this result to integro-partial differential equations. The pioneer result on BSDEs, established by Pardoux and Peng (1990) require Lipschitz condition on the drift of the equation. Sow study on BSDE with jumps, established by Sow (2014) require non-Lipschitz coefficients and application to large deviations.

Mathematical mean-field approaches play an important role in many fields, among them, finance and game theory. Since the pioneering of Lasry and Lions (2007) the research on mean-field has attracted a lot of researchers. Buckdahn, Djehiche, Li and Peng (2009) studied a type of mean field problem by a purely stochastic approach and introduced a new type of BSDE which they called mean-field BSDE. Buckdahn, Li and Peng (2009) obtained the existence and the uniqueness of the solution of the mean-field BSDEs when the coefficient $f$ is Lipschitz, and the terminal condition $\xi$ is a square integrable random variable. They also got a comparison theorem. Later, more and more works have been studied on mean-field SDEs and BSDEs, see Buckdahn, Li and Peng (2009), Buckdahn, Li, Peng and Rainer (2017), Hao and Li (2016), Li (2017), Li and Min (2016). Du, Li and Wei (2011) considered a special type of one dimensional mean-field BSDEs with coefficients which are continuous and have a linear growth. They got the existence of the minimal solution. Recently, Juan (2018) considered general mean-field BSDEs with continuous coefficients. Our aim in the present work is to extend result to generalized mean-field BSDEs driven by fractional Brownian motion with continuous coefficients.

Let us recall that, for $H \in(0,1)$, a $\mathrm{fBm}\left(B^{H}(t)\right)_{t \geqslant 0}$ with Hurst parameter $H$ is a continuous and centered Gaussian process with covariance

$$
E\left[B^{H}(t) B^{H}(s)\right]=\frac{1}{2}\left(t^{2 H}+s^{2 H}-|t-s|^{2 H}\right), \quad t, s \geqslant 0 .
$$

For $H=1 / 2$, the $\mathrm{fBm}$ is a standard Brownian motion. If $H>1 / 2$, then $B^{H}(t)$ has a long-range dependence, which means 
that for $r(n):=\operatorname{cov}\left(B^{H}(1), B^{H}(n+1)-B^{H}(n)\right)$, we have $\sum_{n=1}^{\infty} r(n)=\infty$. Moreover, $B^{H}$ is self-similar, i.e. $B^{H}(a t)$ has the same law as $a^{H} B^{H}(t)$ for any $a>0$. Since there are many models of physical phenomena and finance which exploit the self-similarily and the long-range dependence, $\mathrm{fBm}$ are a very useful tool to characterize such type of problems.

However, since $\mathrm{fBm}$ are not semimartingales nor Markov processes when $H \neq 1 / 2$, we can not use the classical theory of stochastic calculus to define the fractional stochastic integral. In essence, two different integration theories with respect to fractional Brownian motion have been defined and studied. The first one, originally due to Young (1936), concerns the pathwise Riemann-Stieljes integral which exists if the integrand has Hölder continuous paths of order $\alpha>1-H$. But it turn out that this integral has the properties comparable to the Stratonovich integral, which leads to difficulties in applications. The second one concerns the divergence operator (Skorohod integral), define as the adjoint of the derivative operator in the framework of the Malliavin calculus. This approach was introduced by Decreusefond and Us̈tun̈el (1998).

Concerning the study of BSDEs in the fractional framework, the major problem is the absence of a martingale representation type theorem with respect to fBm. For the first time, Hu and Peng (2009) overcome this problem, in the case $H>1 / 2$.

We now introduce a class of reflected diffusion processes with standard Brownian motion. Let $G$ be an open connected subset of $R^{d}$, which is such that for some $l \in C^{2}\left(\mathbb{R}^{d}\right), G=\{x: l(x)>0\}, \partial G=\{x: l(x)=0\}$ and $|\nabla l(x)|=1$ for $x \in \partial G$. Note that at any boundary point $x \in \partial G, \nabla l(x)$ is a unit normal vector to the boundary, pointing towards to the interior of $G$. If drift coeffcient and diffusion coeffcient satisfying some Lipschitz, then it follows from the results in Lions and Sznitman (1984) (see also Saisho (1987)) that for each $x \in \partial G$, there exists a unique pair of progressively measurable continuous processes $\left(\eta_{t}, \Lambda_{t}\right)$, such that

$$
\begin{gathered}
\eta_{t}=\eta_{0}+\int_{0}^{t} b(s) d s+\int_{0}^{t} \sigma(s) d B_{s}+\int_{0}^{t} \nabla l\left(\eta_{s}\right) d \Lambda_{s}, \quad 0 \leqslant t \leqslant T, \\
\Lambda_{t}=\int_{0}^{t} \mathbf{1}_{\eta_{s} \in \partial G} d \Lambda_{s}, \quad \Lambda . \text { is a nondecreasing process. }
\end{gathered}
$$

the existence of such a problem driven by fBm was shown in Ferrante and Rovira (2013) and a set $D=(0,+\infty)$.

In this paper we study the generalized mean-field BSDEs driven by $\mathrm{fBm}$ with Hurst parameter $H>1 / 2$. We prove that kind of equation has an adapted solution under continuous coefficients. The paper is organized as follows. In section 2 we give some definitions and results about fractional stochastic integral which will be needed throughout the paper. Section 3 contains the definition of the generalized BSDEs driven by $\mathrm{fBm}$ and assumptions. In section 4 , we will prove comparison theorem for the generalized mean-field FBSDE. Finally, section 5 is devoted to prove the main theorem of the paper.

\section{Fractional Stochastic Calculus}

Denote, for given $H \in(1 / 2,1), \phi(x)=H(2 H-1)|x|^{2 H-2}, x \in \mathbb{R}$. Let $\xi$ and $\eta$ be measurable functions on $[0, T]$. Define

$$
\langle\xi, \eta\rangle_{t}=\int_{0}^{t} \int_{0}^{t} \phi(u-v) \xi(u) \eta(v) d u d v
$$

and $\|\xi\|_{t}^{2}=\langle\xi, \xi\rangle_{t}$. Note that , for any $t \in[0, T],\langle\xi, \eta\rangle_{t}$ is a Hilbert scalar product.

Let $\mathcal{H}$ be the completion of the measurable functions such that $\|\xi\|_{t}^{2}<\infty$. The elements of $\mathcal{H}$ may be distributions (refer to Pipiras and Taqqu (2000)).

Let $\left(\xi_{n}\right)_{n}$ be a sequence in $\mathcal{H}$ such that $\left\langle\xi_{i}, \xi_{j}\right\rangle_{T}=\delta_{i j}$. By $\mathcal{P}_{T}$ denote the set of all polynomials of fractional Brownian motion in $[0, T]$, i.e. it contains all elements of the form

$$
F(\omega)=f\left(\int_{0}^{T} \xi_{1}(t) d B_{t}^{H}, \cdots, \int_{0}^{T} \xi_{k}(t) d B_{t}^{H}\right),
$$

where $f$ is a polynomial function of $k$ variables. The Malliavin derivative operator $D_{s}^{H}$ of an element $F \in \mathcal{P}_{T}$ is defined as follows:

$$
D_{s}^{H} F=\sum_{i=1}^{k} \frac{\partial f}{\partial x_{i}}\left(\int_{0}^{T} \xi_{1}(t) d B_{t}^{H}, \cdots, \int_{0}^{T} \xi_{k}(t) d B_{t}^{H}\right) \cdot \xi_{i}(s), \quad s \in[0, T] .
$$

Since the divergence operator $D^{H}$ is closable from $L^{2}(\Omega, \mathcal{F}, P)$ to $(\Omega, \mathcal{F}, \mathcal{H})$, By $\mathbb{D}_{1,2}$ denote the Banach space be the a completion of $\mathcal{P}_{T}$ with the following norm: $\|F\|_{1,2}^{2}=E|F|^{2}+E\left\|D_{s}^{H} F\right\|_{T}^{2}$. 
Now we also introduce another derivative

$$
\mathbb{D}_{t}^{H} F=\int_{0}^{T} \phi(t-s) D_{s}^{H} F d s
$$

The following results are well known, refer to Duncan and $\mathrm{Hu}$ (2000), $\mathrm{Hu}(2005)$.

Theorem 2.1. (Hu (2005),Proposition 6.25) Let $F:(\Omega, \mathcal{F}, P) \rightarrow \mathcal{H}$ be a stochastic process such that

$$
E\left(\|F\|_{T}^{2}+\int_{0}^{T} \int_{0}^{T}\left|\mathbb{D}_{s}^{H} F_{t}\right|^{2} d s d t\right)<\infty
$$

Then, the Itô-type stochastic integral denoted by $\int_{0}^{T} F_{s} d B_{s}^{H}$ exists in $L^{2}(\Omega, \mathcal{F}, P)$. Moreover, $E\left(\int_{0}^{T} F_{s} d B_{s}^{H}\right)=0$ and

$$
E\left(\int_{0}^{T} F_{s} d B_{s}^{H}\right)^{2}=E\left(\|F\|_{T}^{2}+\int_{0}^{T} \int_{0}^{T} \mathbb{D}_{s}^{H} F_{t} \mathbb{D}_{t}^{H} F_{s} d s d t\right) .
$$

Theorem 2.2. (Hu (2005),Proposition 10.3) Let $f, g:[0, T] \rightarrow \mathbb{R}$ be deterministic continuous functions. If

$$
X_{t}=X_{0}+\int_{0}^{t} g(s) d s+\int_{0}^{t} f(s) d B_{s}^{H}, \quad t \in[0, T],
$$

where $X_{0}$ is a constant and $F \in C^{1,2}([0, T] \times \mathbb{R})$, then

$$
F\left(t, X_{t}\right)=F\left(0, X_{0}\right)+\int_{0}^{t} \frac{\partial F}{\partial s}\left(s, X_{s}\right) d s+\int_{0}^{t} \frac{\partial F}{\partial x}\left(s, X_{s}\right) d X_{s}+\frac{1}{2} \int_{0}^{t} \frac{\partial^{2} F}{\partial x^{2}}\left(s, X_{s}\right) \frac{d}{d s}\left(\|f\|_{s}^{2}\right) d s, \quad t \in[0, T] .
$$

Theorem 2.3. (Hu (2005),Proposition 11.1) Let $f_{i}(s), g_{i}(s), i=1,2$ are in $\mathbb{D}_{1,2}$ and $E \int_{0}^{T}\left(\left|f_{i}(s)\right|+\left|g_{i}(s)\right|\right) d s<\infty$. Assume that $\mathbb{D}_{t}^{H} f_{1}(s)$ and $\mathbb{D}_{t}^{H} f_{2}(s)$ are continuously differential with respect to $(s, t) \in[0, T] \times[0, T]$ for almost all $\omega \in \Omega$. Suppose that

$$
E\left(\int_{0}^{T} \int_{0}^{T}\left|\mathbb{D}_{t}^{H} f_{i}(s)\right|^{2} d s d t\right)<\infty
$$

For $i=1,2$, denote

$$
X_{i}(t)=\int_{0}^{t} g_{i}(s) d s+\int_{0}^{t} f_{i}(s) d B_{s}^{H}, \quad t \in[0, T]
$$

Then

$$
\begin{aligned}
X_{1}(t) X_{2}(t) & =\int_{0}^{t} X_{1}(s) g_{2}(s) d s+\int_{0}^{t} X_{1}(t) f_{2}(s) d B_{s}^{H}+\int_{0}^{t} X_{2}(s) g_{1}(s) d s+\int_{0}^{t} X_{2}(t) f_{1}(s) d B_{s}^{H} \\
& +\int_{0}^{t} \mathbb{D}_{s}^{H} X_{1}(s) f_{2}(s) d s+\int_{0}^{t} \mathbb{D}_{s}^{H} X_{2}(s) f_{1}(s) d s .
\end{aligned}
$$

\section{Generalized Fractional BSDE}

Let $\left(\Omega, \mathcal{F},\left(\mathcal{F}_{t}\right)_{0 \leqslant t \leqslant T}, P\right), T>0$, be a complete stochastic basis, and $\mathcal{F}_{t^{+}}=\cap_{\delta>0} \mathcal{F}_{t+\delta}=\mathcal{F}_{t}$. Suppose that the filtration is generated by $d$-dimensional fractional Brownian motion $\left(B_{t}^{H}\right)_{0 \leqslant t \leqslant T}$, and $T>0$ is an arbitrarily fixed time horizon. We suppose that there is a sub- $\sigma$-field $\mathcal{F}_{0} \subset \mathcal{F}, \mathcal{F}_{0}$ includes all $P$-null subsets of $\mathcal{F}$, such that

i) the fractional Brownian motion $B^{H}$ is independent of $\mathcal{F}_{0}$, and

ii) $\mathcal{F}_{0}$ is "rich enough", i.e., $\mathcal{P}_{2}\left(\mathbb{R}^{k}\right)=\left\{P_{\vartheta}, \vartheta \in L^{2}\left(\mathcal{F}_{0} ; \mathbb{R}^{k}\right)\right\}, k \geqslant 1$.

Recall that $\mathcal{P}_{2}\left(\mathbb{R}^{k}\right)$ is the set of the probability measures on $\left(\mathbb{R}^{k}, B\left(\mathbb{R}^{k}\right)\right)$ with finite second moment. Here $B\left(\mathbb{R}^{k}\right)$ denotes the Borel $\sigma$-field over $\mathbb{R}^{k}$. By $\mathcal{F}=\left(\mathcal{F}_{t}\right), t \in[0, T]$, we denote the filtration generated by $B^{H}$, completed and augmented by $\mathcal{F}_{0}$. 
The space $\mathcal{P}_{2}\left(\mathbb{R}^{d}\right)$ is endowed with the 2 -Wasserstein metric

$$
W_{2}(\mu, v)=\inf _{\pi \in \Pi(\mu, \nu)}\left\{\left(\int_{\mathbb{R}^{d} \times \mathbb{R}^{d}}|x-y|^{2} \pi(d x, d y)\right)^{1 / 2}\right\},
$$

where $\Pi(\mu, v)$ is the family of all couplings of $\mu$ and $v$, i.e., $\pi \in \Pi(\mu, v)$ if and only if $\pi$ is a measure on $\mathbb{R}^{d} \times \mathbb{R}^{d}$ with marginals $\mu, v \in \mathcal{P}_{2}\left(\mathbb{R}^{d}\right)$.

Assume that

- $\eta_{0}$ is a given constant;

- $b, \sigma:[0, T] \rightarrow \mathbb{R}$ are continuous deterministic, $\sigma$ is differentiable and $\sigma_{t} \neq 0, t \in[0, T]$.

Note that, since $\|\sigma\|_{t}^{2}=H(2 H-1) \int_{0}^{t} \int_{0}^{t}|u-v|^{2 H-2} \sigma(u) \sigma(v) d u d v$, we have

$$
\frac{d}{d t}\left(\|\sigma\|_{t}^{2}\right)=2 \sigma(t) \widehat{\sigma}(t)>0, \quad \text { where } \quad \widehat{\sigma}(t)=\int_{0}^{t} \phi(t-v) \sigma(v) d v, \quad 0 \leqslant t \leqslant T .
$$

Remark 3.1. (Remark 6 by Maticiuc and Nie (2015))

There exists a suitable constant $M>0$ which is only dependent $H$ such that

$$
\frac{t^{2 H-1}}{M} \leqslant \frac{\widehat{\sigma}(t)}{\sigma(t)} \leqslant M t^{2 H-1}, \quad 0 \leqslant t \leqslant T .
$$

since

$$
\begin{aligned}
\widehat{\sigma}(t) & =\int_{0}^{t} \phi(t-v) \sigma(v) d v=H(2 H-1) \int_{0}^{t}(t-v)^{2 H-2} \sigma(v) d v=H(2 H-1) \int_{0}^{1}(t(1-u))^{2 H-2} \sigma(t u) t d u \\
& =H(2 H-1) t^{2 H-1} \int_{0}^{1}(1-u)^{2 H-2} \sigma(t u) d u,
\end{aligned}
$$

then by continuity of $\sigma$, we get the remark.

We now introduce a class of reflected processes. Let $G$ be an open connected subset of $\mathbb{R}^{d}$, which is such that for some $l \in C^{2}\left(\mathbb{R}^{d}\right), G=\{x: l(x)>0\}, \partial G=\{x: l(x)=0\}$ and $|\nabla l(x)|=1$ for $x \in \partial G$. Note that at any boundary point $x \in \partial G$, $\nabla l(x)$ is a unit normal vector to the boundary, pointing towards to the interior of $G$. Let $\eta_{0} \in G$ and $\left(\eta_{t}, \Lambda_{t}\right)$ be a solution of the following reflected SDE with respect to fractional Brownian motion

$$
\eta_{t}=\eta_{0}+\int_{0}^{t} b(s) d s+\int_{0}^{t} \sigma(s) d B_{s}^{H}+\int_{0}^{t} \nabla l\left(\eta_{s}\right) d \Lambda_{s}, \quad 0 \leqslant t \leqslant T,
$$

By a solution of (1), we mean a pair of processes such that $\eta$. $\in G, \Lambda$ is a nondecreasing process, $\Lambda_{0}=0$, and $\int_{0}^{T}\left(\eta_{t}-\right.$ a)d $d \Lambda_{s} \leqslant 0$ for any $a \in G$,

$$
\Lambda_{t}=\int_{0}^{t} \mathbf{1}_{\eta_{s} \in \partial G} d \Lambda_{s}
$$

The existence of such a problem was shown in Lions and Sznitman (2007) for a standard Brownian motion.

Remark 3.2. This problem is solved in Ferrante and Rovira (2009) for a fractional Brownian motion and a set $G=(0, \infty)$.

Given a final time $T>0$, a final condition $\xi$, which is a $\mathcal{F}_{T}$ measurable real valued random variable and the functions

$$
f:[0, T] \times \Omega \times \mathbb{R} \times \mathcal{P}_{2}\left(\mathbb{R}^{1+d}\right) \times \mathbb{R} \times \mathbb{R}^{d} \rightarrow \mathbb{R}, \quad g:[0, T] \times \Omega \times \mathbb{R} \times \mathcal{P}_{2}(\mathbb{R}) \times \mathbb{R} \rightarrow \mathbb{R},
$$

we consider the following generalized BSDE with respect to $\mathrm{BBm}$ with parameters $(\xi, f, g, \Lambda)$ (short name GFBSDE) whose generators depend on both the solution $(Y, Z)$ and the law of $(Y, Z)$, the law of $Y$, respectivity, i.e.

$$
Y_{t}=\xi+\int_{t}^{T} f\left(s, \eta_{s}, P_{\left(Y_{s}, Z_{s}\right)}, Y_{s}, Z_{s}\right) d s+\int_{t}^{T} g\left(s, \eta_{s}, P_{\left(Y_{s}\right)}, Y_{s}\right) d \Lambda_{s}-\int_{t}^{T} Z_{s} d B_{s}^{H}, \quad 0 \leqslant t \leqslant T .
$$


in order to give a probabilistic formula for the solution of a system of elliptic PDEs, this requires the new term-an integral with respect to a increasing process in this equation (2) which is independent of $Z_{s}$, the local time of the diffusion on the boundary.

Next we introduce the following sets:

- $C_{p o l}^{1,2}([0, T] \times \mathbb{R})$ is the space of all $C^{1,2}$ functions over $[0, T] \times \mathbb{R}$, which together with their derivatives are of polynomial growth,

- $\mathcal{V}_{[0, T]}=\left\{Y=\psi(\cdot, \eta): \psi \in C_{p o l}^{1,2}([0, T] \times \mathbb{R}), \frac{\partial \psi}{\partial t} \quad\right.$ is bounded $\left.\quad, t \in[0, T]\right\}$,

- $\widetilde{\mathcal{V}}_{[0, T]}^{H}$ the completion of $\mathcal{V}_{[0, T]}$ under the following norm (where $\beta>0$ )

$$
\|Y\|_{\beta}=\left(\int_{0}^{T} t^{2 H-1} E\left[e^{\beta \Lambda_{t}}\left|Y_{t}\right|^{2}\right] d t\right)^{1 / 2}=\left(\int_{0}^{T} t^{2 H-1} E\left[e^{\beta \Lambda_{t}}\left|\psi\left(t, \eta_{t}\right)\right|^{2}\right] d t\right)^{1 / 2},
$$

We assume that the coefficients $f$ and $g$ of the GFBSDE are continuous functions and satisfy the following assumption $(H 1)$ :

(H1.1) Linear growth: There exists $K \geqslant 0$, such that

$$
\begin{gathered}
|f(t, \eta, \mu, y, z)| \leqslant K\left(1+W_{2}\left(\mu, \delta_{0}\right)+|y|+|\eta|+|z|\right), d t d P-\text { a.e for all }(\eta, \mu, y, z), \\
|g(t, \eta, v, y)| \leqslant K\left(1+W_{2}\left(v, \delta_{0}\right)+|y|+|\eta|\right), d t d P-\text { a.e for all }(\eta, v, y) .
\end{gathered}
$$

where $\delta_{0}$ is the Dirac measure with mass at $0 \in \mathbb{R}^{1+d}$ or $0 \in \mathbb{R}^{d}$.

(H1.2) Lipschitz in $(\mu, y, z)$ : i.e. there exists a constant $C \in \mathbb{R}^{+}$such that for all $\mu_{1}, \mu_{2} \in \mathcal{P}_{2}\left(\mathbb{R}^{1+d}\right), v_{1}, v_{2} \in \mathcal{P}_{2}\left(\mathbb{R}^{d}\right)$ and all $y_{1}, y_{2} \in \mathbb{R}, z_{1}, z_{2} \in \mathbb{R}^{d}$,

$$
\begin{gathered}
\left|f\left(s, \eta, \mu_{1}, y_{1}, z_{1}\right)-f\left(s, \eta, \mu_{2}, y_{2}, z_{2}\right)\right| \leqslant C\left(W_{2}\left(\mu_{1}, \mu_{2}\right)+\left|y_{1}-y_{2}\right|+\left|z_{1}-z_{2}\right|\right) d s d P-\text { a.e. } \\
\left|g\left(s, \eta, v_{1}, y_{1}\right)-g\left(s, \eta, v_{2}, y_{2}\right)\right| \leqslant C\left(W_{2}\left(v_{1}, v_{2}\right)+\left|y_{1}-y_{2}\right|\right) d s d P-\text { a.e. }
\end{gathered}
$$

(H1.3) A progressively measurable continuous, non-decreasing processes $\Lambda_{t}$ has continuous density function.

(H1.4) There exists $\beta>0$ and a function $\psi$ with bounded derivative such that $\xi=\psi\left(\eta_{T}\right), E\left(e^{\beta \Lambda_{T}}|\xi|^{2}\right)<\infty$ and the integrability condition holds

$$
E\left(\int_{0}^{T} e^{\beta \Lambda_{s}}\left(1+E\left[\left(Y_{s}, Z_{s}\right)^{2}\right]\right) d s+\int_{0}^{T} e^{\beta \Lambda_{s}}\left|\eta_{s}\right|^{2} d s+\int_{0}^{T} e^{\beta \Lambda_{s}}\left(1+E\left[\left(Y_{s}\right)^{2}\right]\right) d \Lambda_{s}\right)<\infty .
$$

\section{Comparison Theorem for General Mean-Field Fractional BSDEs}

Definition 4.1. A binary of processes $\left(Y_{t}, Z_{t}\right)_{0 \leqslant t \leqslant T}$ is called a solution to (2), if $\left(Y_{t}, Z_{t}\right) \in \widetilde{\mathcal{V}}_{[0, T]}^{1 / 2} \times \widetilde{\mathcal{V}}_{[0, T]}^{H}$ and satisfies (2).

Lemma 4.2. Assume $X$ is a mean nonzero Gaussian with nonzero covariance, if for two continuous functions $k_{1}(x), k_{2}(x)$ such that $k_{1}(X)=k_{2}(X)$, then $k_{1}(x)=k_{2}(x)$ for all $x \in \mathbb{R}$.

Proof. Let $f_{X}(x)$ denote the density function of $X$, we have

$$
f_{X}(x)=\frac{1}{\sqrt{2 \pi} \theta} e^{-\frac{(x-\alpha)^{2}}{2 \theta^{2}}}
$$

where $\alpha$ denote mean, $\theta^{2}$ denote variance. Since $k_{1}(X)=k_{2}(X)$, take expectation in both sides of this equality, we get

$$
\int_{-\infty}^{+\infty}\left(k_{1}(x)-k_{2}(x)\right) f_{X}(x) d x=0,
$$

by density of $C_{0}^{\infty}(\mathbb{R})$ in $C(\mathbb{R})$ and $f_{X}(x) \geqslant 0$ for all $x \in \mathbb{R}$, consequently $k_{1}(x)=k_{2}(x)$ for all $x \in \mathbb{R}$.

Lemma 4.3. Assume that $h_{1}, h_{2}$ and $h_{3} \in C_{\text {pol }}^{0,1}([0, T] \times \mathbb{R})$ such that

$$
\int_{0}^{t} h_{1}\left(s, \eta_{s}\right) d s+\int_{0}^{t} h_{2}\left(s, \eta_{s}\right) d B_{s}^{H}+\int_{0}^{t} h_{3}\left(s, \eta_{s}\right) d \Lambda_{s}=0, \quad 0 \leqslant t \leqslant T .
$$

Then we have

$$
h_{1}(s, x)=h_{2}(s, x)=h_{3}(s, x)=0, \quad 0 \leqslant s \leqslant T, \quad x \in \mathbb{R} .
$$


Proof. To simplify notation, we let $\eta_{0}=b(t)=0$ for all $t \in[0, T]$ in (1). Similary to $\mathrm{Hu}$ (2005) Theorem 12.3 , we have

$$
\begin{aligned}
h_{1}\left(s, \eta_{s}\right)= & E h_{1}\left(s, \eta_{s}\right)+\int_{0}^{s}\left(\int_{R} \frac{\partial}{\partial x} p_{u, s}\left(\eta_{u}-y\right) h_{1}\left(s, \eta_{s}\right) d y\right) \sigma(u) d B_{u}^{H} \\
& +\int_{0}^{s}\left(\int_{R} \frac{\partial}{\partial x} p_{u, s}\left(\eta_{u}-y\right) h_{1}\left(s, \eta_{s}\right) d y\right) \nabla l\left(\eta_{u}\right) d \Lambda_{u},
\end{aligned}
$$

where

$$
p_{t}(x)=\frac{1}{\sqrt{2 \pi t}} e^{-\frac{x^{2}}{2 t}}
$$

and

$$
p_{u, s}(x)=p_{\|\sigma\|_{s}-\|\sigma\|_{u}}(x)
$$

Thus, by stochastic Fubini theorem

$$
\begin{aligned}
\int_{0}^{t} h_{1}\left(s, \eta_{s}\right) d s= & \int_{0}^{t} E h_{1}\left(s, \eta_{s}\right) d s+\int_{0}^{t} \int_{0}^{s}\left(\int_{R} \frac{\partial}{\partial x} p_{u, s}\left(\eta_{u}-y\right) h_{1}\left(s, \eta_{s}\right) d y\right) \sigma(u) d B_{u}^{H} d s \\
& +\int_{0}^{t} \int_{0}^{s}\left(\int_{R} \frac{\partial}{\partial x} p_{u, s}\left(\eta_{u}-y\right) h_{1}\left(s, \eta_{s}\right) d y\right) \nabla l\left(\eta_{u}\right) d \Lambda_{u} d s \\
= & \int_{0}^{t} E h_{1}\left(s, \eta_{s}\right) d s+\int_{0}^{t} \sigma(u)\left(\int_{u}^{t} \int_{R} \frac{\partial}{\partial x} p_{u, s}\left(\eta_{u}-y\right) h_{1}\left(s, \eta_{s}\right) d y d s\right) d B_{u}^{H} \\
& +\int_{0}^{t} \nabla l\left(\eta_{u}\right)\left(\int_{u}^{t} \int_{R} \frac{\partial}{\partial x} p_{u, s}\left(\eta_{u}-y\right) h_{1}\left(s, \eta_{s}\right) d y d s\right) d \Lambda_{u} \\
= & \int_{0}^{t} E h_{1}\left(s, \eta_{s}\right) d s+\int_{0}^{t}\left[h_{2}\left(u, \eta_{u}\right)+\sigma(u)\left(\int_{u}^{t} \int_{R} \frac{\partial}{\partial x} p_{u, s}\left(\eta_{u}-y\right) h_{1}\left(s, \eta_{s}\right) d y d s\right)\right] d B_{u}^{H} \\
& +\int_{0}^{t}\left[h_{3}\left(u, \eta_{u}\right)+\nabla l\left(\eta_{u}\right)\left(\int_{u}^{t} \int_{R} \frac{\partial}{\partial x} p_{u, s}\left(\eta_{u}-y\right) h_{1}\left(s, \eta_{s}\right) d y d s\right)\right] d \Lambda_{u} \\
& -\int_{0}^{t} h_{2}\left(u, \eta_{u}\right) d B_{u}^{H}-\int_{0}^{t} h_{3}\left(u, \eta_{u}\right) d \Lambda_{u},
\end{aligned}
$$

Thus from assumption, we have

$$
\begin{gathered}
\int_{0}^{t} E h_{1}\left(s, \eta_{s}\right) d s=0, \\
\int_{0}^{t}\left[h_{2}\left(u, \eta_{u}\right)+\sigma(u) \int_{u}^{t} \int_{R} \frac{\partial}{\partial x} p_{u, s}\left(\eta_{u}-y\right) h_{1}\left(s, \eta_{s}\right) d y d s\right] d B_{u}^{H}=0, \\
\int_{0}^{t}\left[h_{3}\left(u, \eta_{u}\right)+\nabla l\left(\eta_{u}\right) \int_{u}^{t} \int_{R} \frac{\partial}{\partial x} p_{u, s}\left(\eta_{u}-y\right) h_{1}\left(s, \eta_{s}\right) d y d s\right] d \Lambda_{u}=0 .
\end{gathered}
$$

But $h_{2}\left(u, \eta_{u}\right)+\sigma(u) \int_{u}^{t} \int_{R} \frac{\partial}{\partial x} p_{u, s}\left(\eta_{u}-y\right) h_{1}\left(s, \eta_{s}\right) d y d s$ and $h_{3}\left(u, \eta_{u}\right)+\nabla l\left(\eta_{u}\right) \int_{u}^{t} \int_{R} \frac{\partial}{\partial x} p_{u, s}\left(\eta_{u}-y\right) h_{1}\left(s, \eta_{s}\right) d y d s$ are $\mathcal{F}_{u}$ adapted (since these are a function of $\eta_{u}$ ). So from Theorem12.1 of Hu (2005), we see that

$$
\begin{gathered}
h_{2}\left(u, \eta_{u}\right)+\sigma(u) \int_{u}^{t} \int_{R} \frac{\partial}{\partial x} p_{u, s}\left(\eta_{u}-y\right) h_{1}\left(s, \eta_{s}\right) d y d s=0, \\
h_{3}\left(u, \eta_{u}\right)+\nabla l\left(\eta_{u}\right) \int_{u}^{t} \int_{R} \frac{\partial}{\partial x} p_{u, s}\left(\eta_{u}-y\right) h_{1}\left(s, \eta_{s}\right) d y d s=0 .
\end{gathered}
$$

In our situation, $\left(\eta_{u}, \Lambda_{u}\right)$ is a solution of reflected SDE with respect to fractional Brownian motion

$$
\eta_{u}=\int_{0}^{s} \sigma(u) d B_{u}^{H}+\int_{0}^{s} \nabla l\left(\eta_{u}\right) d \Lambda_{u}, \quad 0 \leqslant u \leqslant s,
$$


where $\Lambda$ is a nondecreasing process, and

$$
\Lambda_{u}=\int_{0}^{s} \mathbf{1}_{\eta_{u} \in \partial G} d \Lambda_{u}
$$

Although $\eta_{u}$ is not center Gaussian process, but by Lemma 4.2, we have

$$
\begin{aligned}
& h_{2}(u, z)+\sigma(u) \int_{u}^{t} \int_{R} \frac{\partial}{\partial x} p_{u, s}\left(\eta_{u}-y\right) h_{1}(s, z) d y d s=0, \\
& h_{3}(u, z)+\nabla l(z) \int_{u}^{t} \int_{R} \frac{\partial}{\partial x} p_{u, s}\left(\eta_{u}-y\right) h_{1}(s, z) d y d s=0 .
\end{aligned}
$$

for all $z \in \mathbb{R}$. Next, the step as same as Lemma 4.2 of $\mathrm{Hu}(2005)$,and consequently $h_{1}(s, z)=0$ for all $0 \leqslant s \leqslant T, z \in \mathbb{R}$. Finally, Bringing $h_{1}(s, z)=0$ into the formulas (3) and (4), $h_{2}(u, z)=0, h_{3}(u, z)=0$ are then an immediate consequence for all $0 \leqslant s \leqslant T, z \in \mathbb{R}$.

It is well known following Lemma (refer to $\mathrm{Hu}(2005)$ ).

Lemma 4.4. Let $\left(Y_{t}, Z_{t}\right)_{0 \leqslant t \leqslant T}$ be a solution of the GFBSDE (2). Then we have the stochastic representation

$$
\mathbb{D}_{t}^{H} Y_{t}=\frac{\widehat{\sigma}(t)}{\sigma(t)} Z_{t}, \quad 0 \leqslant t \leqslant T,
$$

Proposition 4.5. Let $\xi \in L^{2}\left(\Omega, \mathcal{F}_{T} ; \mathbb{R}\right)$. Assume (H1) holds. Then there exists a unique solution of (2). Moreover, for all $t \in[0, T]$,

$$
E\left(e^{\beta \Lambda_{s}}\left|Y_{t}\right|^{2}+\int_{t}^{T} e^{\beta \Lambda_{s}} s^{2 H-1}\left|Z_{s}\right|^{2} d s+\int_{t}^{T} e^{\beta \Lambda_{s}}\left|Y_{s}\right|^{2} d \Lambda_{s}\right) \leqslant K \Theta(t, T),
$$

where

$$
\Theta(t, T):=E\left(e^{\beta \Lambda_{T}}|\xi|^{2}+2 \int_{t}^{T} e^{\beta \Lambda_{s}}\left(1+E\left[\left(Y_{s}, Z_{s}\right)^{2}\right]\right) d s+\int_{t}^{T} e^{\beta \Lambda_{s}}\left|\eta_{s}\right|^{2} d s+2 \int_{t}^{T} e^{\beta \Lambda_{s}}\left(1+E\left[\left(Y_{s}\right)^{2}\right]\right) d \Lambda_{s}\right) .
$$

Proof. First we will show the second part of the above theorem. Assume that $(Y, Z)$ is a solution of (5). By $K$ we will denote a constant which may vary from line to line. From the Itô formula

$$
\begin{aligned}
& e^{\beta \Lambda_{t}}\left|Y_{t}\right|^{2}+2 \int_{t}^{T} e^{\beta \Lambda_{s}}\left(\mathbb{D}_{s}^{H} Y_{s}\right) Z_{s} d s+\beta \int_{t}^{T} e^{\beta \Lambda_{s}}\left|Y_{s}\right|^{2} d \Lambda_{s} \\
= & e^{\beta \Lambda_{T}}|\xi|^{2}+2 \int_{t}^{T} e^{\beta \Lambda_{s}}\left|Y_{s}\right| f\left(s, \eta_{s}, P_{\left(Y_{s}, Z_{s}\right)}, Y_{s}, Z_{s}\right) d s+2 \int_{t}^{T} e^{\beta \Lambda_{s}}\left|Y_{s}\right| g\left(s, \eta_{s}, P_{\left(Y_{s}\right)}, Y_{s}\right) d \Lambda_{s} \\
& +2 \int_{t}^{T} e^{\beta \Lambda_{s}}\left|Y_{s}\right| Z_{s} d B_{s}^{H} .
\end{aligned}
$$

By linear growth of $f$ and $g$, for all $\mu \in \mathcal{P}_{2}\left(\mathbb{R}^{1+d}\right), v \in \mathcal{P}_{2}\left(\mathbb{R}^{d}\right)$, we have

$$
\begin{aligned}
& 2|y f(t, \eta, \mu, y, z)| \leqslant 2 K|y|\left(1+W_{2}\left(\mu, \delta_{0}\right)+|\eta|+|y|+|z|\right) \\
& \leqslant\left(2 K^{2}+2 K+\frac{M K^{2}}{s^{2 H-1}}\right)|y|^{2}+|\eta|^{2}+\frac{1}{M} s^{2 H-1}|z|^{2}+\left(1+W_{2}\left(\mu, \delta_{0}\right)\right)^{2} \\
& 2|y g(t, \eta, v, y)| \leqslant 2 K|y|\left(1+W_{2}\left(v, \delta_{0}\right)+|\eta|+|y|\right) \leqslant\left(2 K+2 K^{2}\right)|y|^{2}+|\eta|^{2}+\left(1+W_{2}\left(v, \delta_{0}\right)\right)^{2}
\end{aligned}
$$

There, we can write

$$
\begin{aligned}
& E\left(e^{\beta \Lambda_{t}}\left|Y_{t}\right|^{2}+\frac{2}{M} \int_{t}^{T} e^{\beta \Lambda_{s}} s^{2 H-1}\left|Z_{s}\right|^{2} d s+\beta \int_{t}^{T} e^{\beta \Lambda_{s}}\left|Y_{s}\right|^{2} d \Lambda_{s}\right) \\
\leqslant & E\left(e^{\beta \Lambda_{T}}|\xi|^{2}\right)+2 E \int_{t}^{T} e^{\beta \Lambda_{s}}\left|Y_{s}\right| f\left(s, \eta_{s}, P_{\left(Y_{s}, Z_{s}\right)}, Y_{s}, Z_{s}\right) d s+2 E \int_{t}^{T} e^{\beta \Lambda_{s}}\left|Y_{s}\right| g\left(s, \eta_{s}, P_{\left(Y_{s}\right)}, Y_{s}\right) d \Lambda_{s}
\end{aligned}
$$




$$
\begin{aligned}
& \leqslant E\left(e^{\beta \Lambda_{T}}|\xi|^{2}\right)+E \int_{t}^{T}\left(2 K^{2}+2 K+\frac{M K^{2}}{s^{2 H-1}}+1\right) e^{\beta \Lambda_{s}}\left|Y_{s}\right|^{2} d s+\left(2 K+2 K^{2}\right) E \int_{t}^{T} e^{\beta \Lambda_{s}}\left|Y_{s}\right|^{2} d \Lambda_{s} \\
& +E \int_{t}^{T} e^{\beta \Lambda_{s}}\left(\left|\eta_{s}\right|^{2}\right) d s+\frac{1}{M} E \int_{t}^{T} s^{2 H-1} e^{\beta \Lambda_{s}}\left|Z_{s}\right|^{2} d s \\
& +E \int_{t}^{T} e^{\beta \Lambda_{s}}\left(1+W_{2}\left(P_{\left(Y_{s}, Z_{s}\right)}, \delta_{0}\right)\right)^{2} d s+E \int_{t}^{T} e^{\beta \Lambda_{s}}\left(1+W_{2}\left(P_{\left(Y_{s}\right)}, \delta_{0}\right)\right)^{2} d \Lambda_{s} \\
& \leqslant \Theta(t, T)+E \int_{t}^{T}\left(2 K^{2}+2 K+\frac{M K^{2}}{s^{2 H-1}}\right) e^{\beta \Lambda_{s}}\left|Y_{s}\right|^{2} d s+\left(2 K+2 K^{2}\right) E \int_{t}^{T} e^{\beta \Lambda_{s}}\left|Y_{s}\right|^{2} d \Lambda_{s} \\
& +\frac{1}{M} E \int_{t}^{T} s^{2 H-1} e^{\beta \Lambda_{s}}\left|Z_{s}\right|^{2} d s
\end{aligned}
$$

Choosing $\beta \geqslant\left(2 K+2 K^{2}+1\right)$, we get

$$
\begin{aligned}
& E\left(e^{\beta \Lambda_{t}}\left|Y_{t}\right|^{2}+\frac{1}{M} \int_{t}^{T} e^{\beta \Lambda_{s}} s^{2 H-1}\left|Z_{s}\right|^{2} d s+\int_{t}^{T} e^{\beta \Lambda_{s}}\left|Y_{s}\right|^{2} d \Lambda_{s}\right) \\
\leqslant & \Theta(t, T)+E \int_{t}^{T}\left(2 K^{2}+2 K+\frac{M K^{2}}{s^{2 H-1}}\right) e^{\beta \Lambda_{s}}\left|Y_{s}\right|^{2} d s .
\end{aligned}
$$

By Gronwall's inequality,

$$
E e^{\beta \Lambda_{t}}\left|Y_{t}\right|^{2} \leqslant \Theta(t, T) \exp \left\{\left(2 K^{2}+2 K\right)(T-t)+M K^{2} \frac{T^{2 H-1}-t^{2 H-1}}{2-2 H}\right\}
$$

and also get

$$
E\left(\int_{t}^{T} e^{\beta \Lambda_{s}} s^{2 H-1}\left|Z_{s}\right|^{2} d s+\int_{t}^{T} e^{\beta \Lambda_{s}}\left|Y_{s}\right|^{2} d \Lambda_{s}\right) \leqslant C \Theta(t, T) .
$$

Now we will prove the existence and uniqueness of the solution of (5). The method used here is the fixed point theorem. We will show that the mapping $\Gamma: \widetilde{\mathcal{V}}_{[0, T]}^{1 / 2} \times \widetilde{\mathcal{V}}_{[0, T]}^{H} \rightarrow \widetilde{\mathcal{V}}_{[0, T]}^{1 / 2} \times \widetilde{\mathcal{V}}_{[0, T]}^{H}$ given by $(X, W) \rightarrow \Gamma(X, W)=(Y, Z)$ is a contraction, where $(Y, Z)$ is a solution of the following generalized BSDE:

$$
Y_{t}=\xi+\int_{t}^{T} f\left(s, \eta_{s}, P_{\left(X_{s}, W_{s}\right)}, X_{s}, W_{s}\right) d s+\int_{t}^{T} g\left(s, \eta_{s}, P_{\left(X_{s}\right)}, X_{s}\right) d \Lambda_{s}-\int_{t}^{T} Z_{s} d B_{s}^{H}
$$

Let $k \in \mathbb{N}$ and $t_{i}=\frac{i-1}{k} T, i=1, \cdots, k+1$. First we will show that $\Gamma$ is a contraction on $\widetilde{\mathcal{V}}_{\left[t_{k}, T\right]}^{1 / 2} \times \widetilde{\mathcal{V}}_{\left[t_{k}, T\right]}^{H}$. Take $X, X^{\prime} \in \widetilde{\mathcal{V}}_{\left[t_{k}, T\right]}^{1 / 2}$, $W, W^{\prime} \in \widetilde{\mathcal{V}}_{\left[t_{k}, T\right]}^{H}$, let $\Gamma(X, W)=(Y, Z), \Gamma\left(X^{\prime}, W^{\prime}\right)=\left(Y^{\prime}, Z^{\prime}\right)$ and let $\bar{Y}=Y-Y^{\prime}, \bar{Z}=Z-Z^{\prime}, \bar{X}=X-X^{\prime}, \bar{W}=W-W^{\prime}$. From Itô formula, for $t \in\left[t_{k}, T\right]$, we have

$$
\begin{aligned}
& E\left(e^{\beta \Lambda_{t}}\left|\bar{Y}_{t}\right|^{2}+2 \int_{t}^{T} e^{\beta \Lambda_{s}}\left(\mathbb{D}_{s}^{H} \bar{Y}_{s}\right) \bar{Z}_{s} d s+\beta \int_{t}^{T} e^{\beta \Lambda_{s}}\left|\bar{Y}_{s}\right|^{2} d \Lambda_{s}\right) \\
= & 2 E \int_{t}^{T} e^{\beta \Lambda_{s}}\left|\bar{Y}_{s}\right|\left(f\left(s, \eta_{s}, P_{\left(X_{s}, W_{s}\right)}, X_{s}, W_{s}\right)-f\left(s, \eta_{s}, P_{\left(X_{s}^{\prime}, W_{s}^{\prime}\right)}, X_{s}^{\prime}, W_{s}^{\prime}\right)\right) d s \\
+ & 2 E \int_{t}^{T} e^{\beta \Lambda_{s}}\left|\bar{Y}_{s}\right|\left(g\left(s, \eta_{s}, P_{\left(X_{s}\right)}, X_{s}\right)-g\left(s, \eta_{s}, P_{\left(X_{s}^{\prime}\right)}, X_{s}^{\prime}\right)\right) d \Lambda_{s}
\end{aligned}
$$

Note that $2\left|\bar{y}_{s} \| f\left(s, \eta_{s}, \mu_{1}, x_{s}, w_{s}\right)-f\left(s, \eta_{s}, \mu_{2}, x_{s}^{\prime}, w_{s}^{\prime}\right)\right| \leqslant 2 C\left|\bar{y}_{s}\right|\left(\left|\bar{x}_{s}\right|+\left|\bar{w}_{s}\right|+W_{2}\left(\mu_{1}, \mu_{2}\right)\right)$.

$2\left|\bar{y}_{s} \| g\left(s, \eta_{s}, v_{1}, x_{s}\right)-g\left(s, \eta_{s}, v_{2}, x_{s}^{\prime}\right)\right| \leqslant 2 C\left|\bar{y}_{s}\right|\left(W_{2}\left(v_{1}, v_{2}\right)+\left|\bar{x}_{s}\right|\right) \leqslant \frac{C^{2}}{\alpha}\left|\bar{y}_{s}\right|^{2}+2 \alpha\left|\bar{x}_{s}\right|^{2}+2 \alpha W_{2}^{2}\left(v_{1}, v_{2}\right)$ for some $\alpha>0$.

Choose $\beta=\frac{C^{2}}{\alpha}+1$. Then by the Schwartz inequality we obtain

$$
\begin{aligned}
& E\left(e^{\beta \Lambda_{t}}\left|\bar{Y}_{t}\right|^{2}+\frac{2}{M} \int_{t}^{T} e^{\beta \Lambda_{s}} s^{2 H-1}\left|\bar{Z}_{s}\right|^{2} d s+\int_{t}^{T} e^{\beta \Lambda_{s}}\left|\bar{Y}_{s}\right|^{2} d \Lambda_{s}\right) \\
= & \left.2 K E \int_{t}^{T} e^{\beta \Lambda_{s}}\left|\bar{Y}_{s}\left(\left|\bar{X}_{s}\right|+\left|\bar{W}_{s}\right|\right) d s+\alpha E \int_{t}^{T} e^{\beta \Lambda_{s}}\right| \bar{X}_{s}\right|^{2} d \Lambda_{s}
\end{aligned}
$$




$$
\leqslant 2 K \int_{t}^{T}\left(E e^{\beta \Lambda_{s}}\left|\bar{Y}_{s}\right|^{2}\right)^{1 / 2}\left(E e^{\beta \Lambda_{s}}\left(\left|\bar{X}_{s}\right|+\left|\bar{W}_{s}\right|\right)^{2}\right)^{1 / 2} d s+\alpha E \int_{t}^{T} e^{\beta \Lambda_{s}}\left|\bar{X}_{s}\right|^{2} d \Lambda_{s}
$$

Denote $\varphi(t)=\left(E e^{\beta \Lambda_{s}}\left|\bar{Y}_{s}\right|^{2}\right)^{1 / 2}$ and $\psi(t)=\alpha E \int_{t}^{T} e^{\beta \Lambda_{s}}\left|\bar{X}_{s}\right|^{2} d \Lambda_{s}$ which is nonincrease. Then by above

$$
\varphi(t)^{2} \leqslant 2 K \int_{t}^{T} \varphi(t)\left(E e^{\beta \Lambda_{s}}\left(\left|\bar{X}_{s}\right|+\left|\bar{W}_{s}\right|\right)^{2}\right)^{1 / 2} d s+\psi(t), \quad t \in\left[t_{k}, T\right] .
$$

Applying Lemma 20 in Maticiuc and Nie (1994) to the above inequality we get

$$
\varphi(t) \leqslant \sqrt{2} K \int_{t}^{T}\left(E e^{\beta \Lambda_{s}}\left(\left|\bar{X}_{s}\right|+\left|\bar{W}_{s}\right|\right)^{2}\right)^{1 / 2} d s+\sqrt{\psi(t)}, \quad t \in\left[t_{k}, T\right] .
$$

and therefore for $t \in\left[t_{k}, T\right]$

$$
E e^{\beta \Lambda_{s}}\left|\bar{Y}_{s}\right|^{2} \leqslant 4 K^{2}\left(\int_{t}^{T}\left(E e^{\beta \Lambda_{s}}\left(\left|\bar{X}_{s}\right|+\left|\bar{W}_{s}\right|\right)^{2}\right)^{1 / 2} d s\right)^{2}+2 \psi(t),
$$

Integrate of both sides on $\left[t_{k}, T\right]$ of above inequality, we can compute

$$
\begin{aligned}
\int_{t_{k}}^{T} \varphi(s)^{2} d s & \leqslant 2 \psi\left(t_{k}\right)\left(T-t_{k}\right)+4 K^{2} \int_{t_{k}}^{T}\left(\int_{t}^{T}\left(E e^{\beta \Lambda_{s}}\left(\left|\bar{X}_{s}\right|+\left|\bar{W}_{s}\right|\right)^{2}\right)^{1 / 2} d s\right)^{2} d t \\
& \leqslant 2 \psi\left(t_{k}\right)\left(T-t_{k}\right)+8 K^{2}\left(T-t_{k}\right)\left(\int_{t_{k}}^{T}\left(E e^{\beta \Lambda_{s}}\left|\bar{X}_{s}\right|^{2}\right)^{1 / 2} d s\right)^{2} \\
& +8 K^{2}\left(T-t_{k}\right)\left(\int_{t_{k}}^{T}\left(\frac{1}{s^{2 H-1}} E e^{\beta \Lambda_{s}} s^{2 H-1}\left|\bar{W}_{s}\right|^{2}\right)^{1 / 2} d s\right)^{2} \\
& \leqslant 2 \psi\left(t_{k}\right)\left(T-t_{k}\right)+8 K^{2}\left(T-t_{k}\right)^{2} E \int_{t_{k}}^{T} e^{\beta \Lambda_{s}}\left|\bar{X}_{s}\right|^{2} d s \\
& +8 K^{2}\left(T-t_{k}\right) \int_{t_{k}}^{T} \frac{1}{s^{2 H-1}} d s E \int_{t_{k}}^{T} e^{\beta \Lambda_{s}} s^{2 H-1}\left|\bar{W}_{s}\right|^{2} d s \\
& :=C \cdot\left(T-t_{k}\right) \widetilde{\Theta}\left(t_{k}, T\right),
\end{aligned}
$$

and similarly

$$
\int_{t_{k}}^{T} \frac{1}{s^{2 H-1}} \varphi(s)^{2} d s \leqslant \frac{C}{2-2 H} \cdot\left(T^{2-2 H}-t_{k}^{2-2 H}\right) \cdot \widetilde{\Theta}\left(t_{k}, T\right),
$$

where

$$
\widetilde{\Theta}\left(t_{k}, T\right)=E\left(\int_{t_{k}}^{T} e^{\beta \Lambda_{s}} s^{2 H-1}\left|\bar{W}_{s}\right|^{2} d s+\int_{t_{k}}^{T} e^{\beta \Lambda_{s}}\left|\bar{X}_{s}\right|^{2}\left(d s+d \Lambda_{s}\right)\right) .
$$

Using above inequalities, we deduce

$$
\begin{aligned}
& E\left(\int_{t_{k}}^{T} e^{\beta \Lambda_{s}} s^{2 H-1}\left|\bar{Z}_{s}\right|^{2} d s+\int_{t_{k}}^{T} e^{\beta \Lambda_{s}}\left|\bar{Y}_{s}\right|^{2}\left(d s+d \Lambda_{s}\right)\right) \\
\leqslant & E \int_{t_{k}}^{T} e^{\beta \Lambda_{s}}\left|\bar{Y}_{s}\right|^{2} d s+C \alpha E \int_{t_{k}}^{T} e^{\beta \Lambda_{s}}\left|\bar{X}_{s}\right|^{2} d \Lambda_{s}+C E \int_{t_{k}}^{T} e^{\beta \Lambda_{s}} \frac{1}{\alpha}\left|\bar{Y}_{s}\right|^{2}\left(2+\frac{1}{s^{2 H-1}}\right) d s \\
+ & C E \int_{t_{k}}^{T} e^{\beta \Lambda_{s}} \alpha\left(\left|\bar{X}_{s}\right|^{2}+s^{2 H-1}\left|\bar{W}_{s}\right|^{2}\right) d s \\
\leqslant & C \cdot\left(T-t_{k}\right) \widetilde{\Theta}\left(t_{k}, T\right)+\frac{C}{\alpha} \int_{t_{k}}^{T} \varphi(s)\left(2+\frac{1}{s^{2 H-1}}\right) d s+C \alpha \widetilde{\Theta}\left(t_{k}, T\right) \\
\leqslant & \left.C\left(\alpha+\left(2+\frac{1}{\alpha}\right)\left(T-t_{k}\right)\right)+\frac{1}{\alpha}\left(T^{2-2 H}-t_{k}^{2-2 H}\right)\right) \widetilde{\Theta}\left(t_{k}, T\right)
\end{aligned}
$$


Choosing $\alpha$ such that $C \alpha \leqslant 1 / 4$ and taking $k$ large enough that $C(\alpha+2)\left(T-t_{k}\right) / \alpha \leqslant 1 / 4$ and $C\left(T^{2-2 H}-t_{k}^{2-2 H}\right) / \alpha \leqslant 1 / 4$, we obtain

$$
\begin{aligned}
& E\left(\int_{t_{k}}^{T} e^{\beta \Lambda_{s}} s^{2 H-1}\left|\bar{Z}_{s}\right|^{2} d s+\int_{t_{k}}^{T} e^{\beta \Lambda_{s}}\left|\bar{Y}_{s}\right|^{2}\left(d s+d \Lambda_{s}\right)\right) \\
\leqslant & \frac{3}{4} \widetilde{\Theta}\left(t_{k}, T\right)
\end{aligned}
$$

Thus $\Gamma$ is contraction operator in $\widetilde{\mathcal{V}}_{\left[t_{k}, T\right]}^{1 / 2} \times \widetilde{\mathcal{V}}_{\left[t_{k}, T\right]}^{H}$, and $\left(Y^{m}, Z^{m}\right)$ is a Cauchy sequence in $\widetilde{\mathcal{V}}_{\left[t_{k}, T\right]}^{1 / 2} \times \widetilde{\mathcal{V}}_{\left[t_{k}, T\right]}^{H}$, where $\left(Y^{0}, Z^{0}\right) \in$ $\widetilde{\mathcal{V}}_{\left[t_{k}, T\right]}^{1 / 2} \times \widetilde{\mathcal{V}}_{\left[t_{k}, T\right]}^{H}$, and for $m \geqslant 0$

$$
\begin{aligned}
Y_{t}^{m+1} & :=\xi+\int_{t}^{T} f\left(s, \eta_{s}, P_{\left(Y_{s}^{m}, Z_{s}^{m}\right)}, Y_{s}^{m}, Z_{s}^{m}\right) d s+\int_{t}^{T} g\left(s, \eta_{s}, P_{\left(Y_{s}^{m}\right)}, Y_{s}^{m}\right) d \Lambda_{s} \\
& -\int_{t}^{T} Z_{s}^{m+1} d B_{s}^{H} .
\end{aligned}
$$

Then there exists $(Y, Z) \in \widetilde{\mathcal{V}}_{\left[t_{k}, T\right]}^{1 / 2} \times \widetilde{\mathcal{V}}_{\left[t_{k}, T\right]}^{H}$ being a limit of $\left(Y^{m}, Z^{m}\right)$, i.e.

$$
\begin{gathered}
\lim _{m \rightarrow+\infty} E\left(e^{\beta \Lambda_{t}}\left|Y_{t}^{m}-Y_{t}\right|^{2}+\int_{t_{k}}^{T} e^{\beta \Lambda_{s}}\left(\left|Y_{s}^{m}-Y_{s}\right|^{2}+s^{2 H-1}\left|Z_{s}^{m}-Z_{s}\right|^{2}\right) d s\right)=0, \\
\lim _{m \rightarrow+\infty} E\left(\int_{t_{k}}^{T} e^{\beta \Lambda_{s}}\left|Y_{s}^{m}-Y_{s}\right|^{2} d \Lambda_{s}\right)=0,
\end{gathered}
$$

Therefore for any $t \in\left[t_{k}, T\right]$,

$$
\begin{aligned}
& \lim _{m \rightarrow \infty}\left(-Y_{t}^{m+1}+\xi+\int_{t}^{T} f\left(s, \eta_{s}, P_{\left(Y_{s}^{m}, Z_{s}^{m}\right)}, Y_{s}^{m}, Z_{s}^{m}\right) d s+\int_{t}^{T} g\left(s, \eta_{s}, P_{\left(Y_{s}^{m}\right)}, Y_{s}^{m}\right) d \Lambda_{s}\right) \\
= & -Y_{t}+\xi+\int_{t}^{T} f\left(s, \eta_{s}, P_{\left(Y_{s}, Z_{s}\right)}, Y_{s}, Z_{s}\right) d s+\int_{t}^{T} g\left(s, \eta_{s}, P_{\left(Y_{s}\right)}, Y_{s}\right) d \Lambda_{s}
\end{aligned}
$$

in $L^{2}(\Omega, \mathcal{F}, P)$ and $Z^{m} \mathbf{1}_{[t, T]} \rightarrow Z \mathbf{1}_{[t, T]}$ in $L^{2}(\Omega, \mathcal{F}, \mathcal{H})$. We show $(Y, Z)$ that satisfies (5) on $\left[t_{k}, T\right]$. The next step is to solve the equation on $\left[t_{k-1}, t_{k}\right]$. With the same arguments, repeating the above technique we obtain a uniqueness of the solution of generalized BSDE with respect to $\mathrm{fBm}$ on the whole interval $[0, T]$.

Now we would like to study the comparison theorem. From the counter examples in Borkowska (2013) (see the example 3.1 and 3.2 therein) and example 2.1 in Juan, Hao and Zhang (2018) (only need to simple modify ), we know that if the driver $f$ depends on the law of $Z$ or is not increasing with respect to the law of $Y$, we usually do not have the comparison theorem. Now we give two examples here.

Example: Let $d=1$. We consider

$$
Y_{t}^{i}=\xi^{i}+\int_{t}^{T} E\left[\left|Z_{s}^{i}\right|\right] d s-\int_{t}^{T} Z_{s}^{i} d B_{s}^{H}, \quad i=1,2 . \quad 0 \leqslant t \leqslant T .
$$

For $\xi^{2}=0,\left(Y^{2}, Z^{2}\right)=(0,0)$, in particular, $Y_{0}^{2}=0$. We consider two cases for $\xi^{1}$.

(i) For $\xi^{1}:=-\left(\left(B_{T}^{H}\right)^{+}\right)^{2} \leqslant 0, Z_{t}^{1}:=E\left[D_{t}^{H}\left[\xi^{1}\right] \mid \mathcal{F}_{t}\right]=-2 E\left[\left(B_{T}^{H}\right)^{+} \mid \mathcal{F}_{t}\right] \leqslant 0$. Thus $E\left[\left|Z_{t}^{1}\right|\right]=E\left[-Z_{t}^{1}\right]=2 E\left[\left(B_{T}^{H}\right)^{+}\right]=$ $2 \int_{0}^{\infty} x \frac{1}{\sqrt{2 \pi} T^{H}} e^{-\frac{x^{2}}{2 T^{2 H}}} d x=\frac{2 T^{H}}{\sqrt{2 \pi}}, t \in[0, T]$. And $Y_{0}^{1}=E\left[\xi^{1}\right]+\int_{t}^{T} E\left[\left|Z_{s}^{1}\right|\right] d s=-\frac{T^{2 H}}{2}+\frac{2 T^{H+1}}{\sqrt{2 \pi}}>0$, for $T>\left(\frac{\sqrt{2 \pi}}{4}\right) \frac{1}{1-H}$, i.e. for $T>\left(\frac{\sqrt{2 \pi}}{4}\right)^{\frac{1}{1-H}}, Y_{0}^{1}>0=Y_{0}^{2}$, although $\xi^{1} \leqslant 0=\xi^{2}, P-$ a.s.

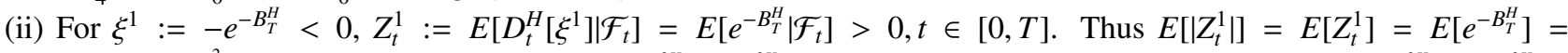
$\int_{R} e^{-x} \frac{1}{\sqrt{2 \pi} T^{H}} e^{-\frac{x^{2}}{2 T^{2 H}}} d x=\frac{1}{\sqrt{2 \pi} T^{H}} \int_{R} e^{-\frac{1}{2 T^{2 H}}\left(x+T^{2 H}\right)^{2}} d x e^{\frac{T^{2 H}}{2}}=e^{\frac{T^{2} H}{2}}, t \in[0, T]$, and $Y_{0}^{1}=E\left[\xi^{1}\right]+\int_{t}^{T} E\left[\left|Z_{s}^{1}\right|\right] d s=-e^{\frac{T^{2 H}}{2}}+T e^{\frac{T^{2 H}}{2}}>$ 0 , for $T>1$, i.e. for $T>1, Y_{0}^{1}>0=Y_{0}^{2}$, although $\xi^{1}<0=\xi^{2}, P-$ a.s.

We consider now the mean-field BSDE as follows

$$
Y_{t}=\xi+\int_{t}^{T} f\left(s, \eta_{s}, P_{Y_{s}}, Y_{s}, Z_{s}\right) d s+\int_{t}^{T} g\left(s, \eta_{s}, P_{Y_{s}}, Y_{s}\right) d \Lambda_{s}-\int_{t}^{T} Z_{s} d B_{s}^{H}, \quad 0 \leqslant t \leqslant T .
$$


Theorem 4.6. (Comparison theorem) Let $\left(f_{i}, g_{i}\right)=\left(f_{i}(s, \omega, \eta, \mu, y, z), g_{i}(s, \omega, \eta, v, y)\right), i=1,2$, be two pair drivers satisfying the assumption (H1.4). Moreover, we suppose

(i) One of the both coefficients pairs satisfies Lipschitz in $(\mu, y, z)$ and $(\nu, y)$.

(ii) One of the both coefficients pairs satisfies: for all $\theta_{1}, \theta_{2} \in L^{2}(\Omega, \mathcal{F} ; \mathbb{R})$, and all $(s, \eta, y, z) \in[0, T] \times \mathbb{R} \times \mathbb{R} \times \mathbb{R}^{d}$, $f_{i}\left(s, \eta, P_{\theta_{1}}, y, z\right)-f_{i}\left(s, \eta, P_{\theta_{2}}, y, z\right) \leqslant L\left(E\left[\left(\left(\theta_{1}-\theta_{2}\right)^{+}\right)^{2}\right]\right)^{1 / 2}$,

$g_{i}\left(s, \eta, P_{\theta_{1}}, y\right)-g_{i}\left(s, \eta, P_{\theta_{2}}, y\right) \leqslant L\left(E\left[\left(\left(\theta_{1}-\theta_{2}\right)^{+}\right)^{2}\right]\right)^{1 / 2}$.

Let $\xi_{1}, \xi_{2} \in L^{2}\left(\Omega, \mathcal{F}_{T} ; \mathbb{R}\right)$ and denote by $\left(Y^{1}, Z^{1}\right)$ and $\left(Y^{2}, Z^{2}\right)$ the solution of the mean-field BSDE $(6)$ with data $\left(\xi_{1}, f_{1}, g_{1}\right)$ and $\left(\xi_{2}, f_{2}, g_{2}\right)$, respectively. Then, if $\xi_{1} \leqslant \xi_{2}, P-a . s ., f_{1}(s, \eta, \mu, y, z) \leqslant f_{2}(s, \eta, \mu, y, z), d s d P-$ a.e., and $g_{1}(s, \eta, v, y) \leqslant$ $g_{2}(s, \eta, v, y), d s d P-$ a.e.for all $(\eta, \mu, v, y, z)$, it holds that also $Y_{s}^{1} \leqslant Y_{s}^{2}$, for all $s \in[0, T], P-$ a.s.

Proof. Without loss of generality, we assume that (i) and (ii) are satisfied by $\left(f_{1}, g_{1}\right)$. Let us put $\bar{f}_{s}:=f_{1}\left(s, \eta, P_{Y_{s}^{1}}, Y_{s}^{1}, Z_{s}^{1}\right)-$ $f_{2}\left(s, \eta, P_{Y_{s}^{2}}, Y_{s}^{2}, Z_{s}^{2}\right), \overline{g_{s}}:=g_{1}\left(s, \eta, P_{Y_{s}^{1}}, Y_{s}^{1}\right)-g_{2}\left(s, \eta, P_{Y_{s}^{2}}, Y_{s}^{2}\right)$, and $\overline{Z_{s}}:=Z_{s}^{1}-Z_{s}^{2}, \overline{Y_{s}}:=Y_{s}^{1}-Y_{s}^{2}$. From Itô-Tanakas formula applied to $\left({\overline{Y_{s}}}^{+}\right)^{2}$, we have

$$
E\left[\left({\overline{Y_{s}}}^{+}\right)^{2}\right]+E \int_{t}^{T} \frac{d}{d s}\left\|\overline{Z_{r}}\right\|_{s}^{2} 1_{\left.\overline{Y_{s}}>0\right)} d s=2 E \int_{t}^{T} \overline{Y_{s}} 1_{\left(\overline{Y_{s}}>0\right)} \overline{f_{s}} d s+2 E \int_{t}^{T} \overline{Y_{s}} 1_{\left.\overline{\left(Y_{s}\right.}>0\right)} \overline{g_{s}} d \Lambda_{s},
$$

Notice that, since $\left(f_{1}, g_{1}\right)$ is Lipschitz continuous and $f_{1} \leqslant f_{2}, g_{1} \leqslant g_{2}$, we have

$$
\begin{aligned}
& E\left[\left({\overline{Y_{s}}}^{+}\right)^{2}\right]+E \int_{t}^{T} \frac{d}{d s}\left(\left\|\overline{Z_{r}}\right\|_{s}^{2}\right) 1_{\left(\overline{Y_{s}}>0\right)} d s \\
\leqslant & 2 E \int_{t}^{T} \bar{Y}_{s}^{+} 1_{\left(\overline{Y_{s}}>0\right)}\left(f_{1}\left(s, \eta, P_{Y_{s}^{1}}, Y_{s}^{1}, Z_{s}^{1}\right)-f_{2}\left(s, \eta, P_{Y_{s}^{2}}, Y_{s}^{1}, Z_{s}^{1}\right)+C\left|\overline{Y_{s}}\right|+C\left|\overline{Z_{s}}\right|\right) d s \\
& +2 E \int_{t}^{T}{\overline{Y_{s}}}^{+} 1_{\left(\overline{Y_{s}}>0\right)}\left(g_{1}\left(s, \eta, P_{Y_{s}^{1}}, Y_{s}^{1}\right)-g_{2}\left(s, \eta, P_{Y_{s}^{2}}, Y_{s}^{1}\right)+C\left|\overline{Y_{s}}\right|\right) d \Lambda_{s},
\end{aligned}
$$

Moreover, as for all $\theta_{1}, \theta_{2} \in L^{2}(\Omega, \mathcal{F} ; \mathbb{R})$ and $(s, \eta, y, z) \in[0, T] \times \mathbb{R} \times \mathbb{R} \times \mathbb{R}^{d}$,

$$
\begin{gathered}
f_{1}\left(s, \eta, P_{\theta_{1}}, y, z\right)-f_{1}\left(s, \eta, P_{\theta_{2}}, y, z\right) \leqslant L\left(E\left[\left(\left(\theta_{1}-\theta_{2}\right)^{+}\right)^{2}\right]\right)^{1 / 2}, \\
g_{1}\left(s, \eta, P_{\theta_{1}}, y\right)-g_{1}\left(s, \eta, P_{\theta_{2}}, y\right) \leqslant L\left(E\left[\left(\left(\theta_{1}-\theta_{2}\right)^{+}\right)^{2}\right]\right)^{1 / 2} .
\end{gathered}
$$

we have

$$
\begin{aligned}
& E\left[\left({\overline{Y_{s}}}^{+}\right)^{2}\right]+E \int_{t}^{T} \frac{d}{d s}\left(\left\|\overline{Z_{r}}\right\|_{s}^{2}\right) 1_{\left(\overline{Y_{s}}>0\right)} d s \\
\leqslant & C E \int_{t}^{T}{\overline{Y_{s}}}^{+} 1_{\left(\overline{Y_{s}}>0\right)}\left(\left(E\left[\left({\overline{Y_{s}}}^{+}\right)^{2}\right]\right)^{1 / 2}+\left|\overline{Y_{s}}\right|+\left|\overline{Z_{s}}\right|\right) d s \\
& +C E \int_{t}^{T}{\overline{Y_{s}}}^{+} 1_{\left(\overline{Y_{s}}>0\right)}\left(\left(E\left[\left({\overline{Y_{s}}}^{+}\right)^{2}\right]\right)^{1 / 2}+\left|\overline{Y_{s}}\right|\right) d \Lambda_{s},
\end{aligned}
$$

by Remark 3.1, we obtain, there exists a suitable constant $M>0$,

$$
\frac{2}{M} s^{2 H-1}\left|\overline{Z_{s}}\right|^{2} \leqslant \frac{d}{d s}\left(\left\|\overline{Z_{r}}\right\|_{s}^{2}\right) \leqslant 2 M s^{2 H-1}\left|\overline{Z_{s}}\right|^{2},
$$

Thus

$$
\begin{aligned}
& E\left[\left(\overline{Y_{s}}\right)^{2}\right]+\frac{2}{M} E \int_{t}^{T} s^{2 H-1}\left|\overline{Z_{s}}\right|^{2} 1_{\left(\overline{Y_{s}}>0\right)} d s \\
\leqslant & C E \int_{t}^{T} \overline{Y_{s}} 1_{\left(\overline{Y_{s}}>0\right)}\left(\left(E\left[\left({\overline{Y_{s}}}^{+}\right)^{2}\right]\right)^{1 / 2}+\left|\overline{Y_{s}}\right|+\left|\overline{Z_{s}}\right|\right) d s \\
& +C E \int_{t}^{T} \overline{Y_{s}} 1_{\left(\overline{Y_{s}}>0\right)}\left(\left(E\left[\left({\overline{Y_{s}}}^{+}\right)^{2}\right]\right)^{1 / 2}+\left|\overline{Y_{s}}\right|\right) d \Lambda_{s} \\
\leqslant & C E \int_{t}^{T}\left({\overline{Y_{s}}}^{+}\right)^{2} d s+C E \int_{t}^{T}\left(\overline{Y_{s}}\right)^{2} s^{1-2 H} d s+C E \int_{t}^{T}\left|\overline{Z_{s}}\right|^{2} s^{2 H-1} 1_{\left(\overline{Y_{s}}>0\right)} d s+C E \int_{t}^{T}\left({\overline{Y_{s}}}^{+}\right)^{2} d \Lambda_{s}
\end{aligned}
$$




$$
\leqslant C E \int_{t}^{T}\left({\overline{Y_{s}}}^{+}\right)^{2}\left(1+p(s)+s^{1-2 H}\right) d s+C E \int_{t}^{T}\left|\overline{Z_{s}}\right|^{2} s^{2 H-1} 1_{\left(\overline{Y_{s}}>0\right)} d s,
$$

the last inequality applies assumption (H1.4). Choose suitable $M$, such that $\frac{2}{M}-C>0$, then we have

$$
E\left[\left({\overline{Y_{s}}}^{+}\right)^{2}\right] \leqslant C E \int_{t}^{T}\left({\overline{Y_{s}}}^{+}\right)^{2}\left(1+p(s)+s^{1-2 H}\right) d s,
$$

From Gronwall's inequality, $E\left({\overline{Y_{s}}}^{+}\right)^{2}=0, s \in[0, T]$, i.e. $Y_{s}^{1} \leqslant Y_{s}^{2}, P-a . s, s \in[0, T]$.

\section{General Mean-Field Fractional BSDEs Under Continuous Coefficients}

We assume that the coefficients $f$ and $g$ of the GFBSDE are continuous functions and satisfy the following assumption $(H 2)$ :

(H2.1) Linear growth: There exists $K \geqslant 0$, such that

$$
\begin{gathered}
|f(t, \eta, \mu, y, z)| \leqslant K\left(1+W_{2}\left(\mu, \delta_{0}\right)+|y|+|\eta|+|z|\right), d t d P-\text { a.e for all }(\eta, \mu, y, z), \\
\qquad|g(t, \eta, v, y)| \leqslant K\left(1+W_{2}\left(v, \delta_{0}\right)+|y|+|\eta|\right), d t d P-\text { a.e for all }(\eta, v, y) .
\end{gathered}
$$

where $\delta_{0}$ is the Dirac measure with mass at $0 \in \mathbb{R}^{1+d}$ or $0 \in \mathbb{R}^{d}$.

(H2.2) Monotonicity in $\mu$ : for all $\theta_{1}, \theta_{2} \in L^{2}(\Omega, \mathcal{F} ; \mathbb{R})$, and all $(\eta, y, z) \in \mathbb{R} \times \mathbb{R} \times \mathbb{R}^{d}$,

$$
\begin{gathered}
f\left(s, \eta, P_{\theta_{2}}, y, z\right) \leqslant f\left(s, \eta, P_{\theta_{1}}, y, z\right), d t d P-a . e, \text { whenever } \theta_{2} \leqslant \theta_{1}, \\
g\left(s, \eta, P_{\theta_{2}}, y\right) \leqslant g\left(s, \eta, P_{\theta_{1}}, y\right), d t d P-a . e, \text { whenever } \theta_{2} \leqslant \theta_{1} .
\end{gathered}
$$

$(\boldsymbol{H 2 . 3})$ For a.e. $(s, \omega) \in[0, T] \times \Omega, f(s, \omega, \cdot, \cdot, \cdot, \cdot), g(s, \omega, \cdot, \cdot, \cdot)$ are continuous with a continuity modulus $\rho: \mathbb{R}^{+} \rightarrow \mathbb{R}^{+}$for $\mu$ :

$$
\left|f\left(s, \omega, \eta, \mu_{1}, y, z\right)-f\left(s, \omega, \eta, \mu_{2}, y, z\right)\right|+\left|g\left(s, \omega, \eta, v_{1}, y\right)-g\left(s, \omega, \eta, v_{2}, y\right)\right| \leqslant \rho\left(W_{2}\left(\mu_{1}, \mu_{2}\right)\right) .
$$

Here $\rho$ is supposed to be increasing and such that $\rho(0+)=0$.

Remark 5.1. (H2.2) is equivalent to the following condition:

(H2.2'): For all $\mu_{1}, \mu_{2} \in \mathcal{P}_{2}(\mathbb{R}),(s, \eta, y, z) \in[0, T] \times \mathbb{R} \times \mathbb{R} \times \mathbb{R}^{d}$, it holds $f\left(s, \eta, \mu_{2}, y, z\right) \leqslant f\left(s, \eta, \mu_{1}, y, z\right)$, whenever the distribution functions $F_{\mu_{1}}, F_{\mu_{2}}$ satisfy $F_{\mu_{1}} \leqslant F_{\mu_{2}}$. Recall that $F_{\mu}(x)=\mu((-\infty, x]), x \in \mathbb{R}, \mu \in \mathcal{P}_{2}(\mathbb{R})$.

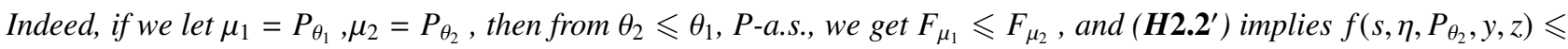
$f\left(s, \eta, P_{\theta_{1}}, y, z\right)$. This shows that $\left(\boldsymbol{H}^{2.2} \mathbf{2}^{\prime}\right) \Rightarrow(\boldsymbol{H 2 . 2})$.

In order to show that $(\boldsymbol{H 2 . 2}) \Rightarrow(\boldsymbol{H 2 . 2})$ : We consider $\mu_{1}, \mu_{2} \in \mathcal{P}_{2}(\mathbb{R})$, with $F_{\mu_{1}} \leqslant F_{\mu_{2}}$. Let $\xi$ be a random variable uniformly distributed on $[0,1]$, and let $F_{\mu_{i}}^{-1}$ be the left inverse function of $F_{\mu_{i}}$. Then $\theta_{2}:=F_{\mu_{2}}^{-1}(\xi) \leqslant F_{\mu_{1}}^{-1}(\xi)=: \theta_{1}$, and $P_{\theta_{1}}=\mu_{1}, P_{\theta_{2}}=\mu_{2}$. From $(\boldsymbol{H 2 . 2})$ we get $f\left(s, \eta, P_{\theta_{2}}, y, z\right) \leqslant f\left(s, \eta, P_{\theta_{1}}, y, z\right)$.

Before proving the main theorem in this paper, we need the following lemma which gives the approximation of continuous functions by the Lipschitz functions and it was presented by Lepeltier and Martin (1997). we have to introduce a new method to study the relationship between two measures, we define

$$
W_{2,+}(\mu, v)=\inf _{\pi \in \Pi(\mu, v)}\left\{\left(\int_{\mathbb{R}^{d} \times \mathbb{R}^{d}}\left|(x-y)^{+}\right|^{2} \pi(d x, d y)\right)^{1 / 2}\right\},
$$

where $\Pi(\mu, v)$ is the family of all couplings of $\mu$ and $v$, i.e., $\pi \in \Pi(\mu, v)$ if and only if $\pi$ is a measure on $\mathbb{R}^{d} \times \mathbb{R}^{d}$ with marginals $\mu, v \in \mathcal{P}_{2}\left(\mathbb{R}^{d}\right)$.

The following Lemma is a modified based on Lemma 3.1 in Li, Liang and Zhang (2018). 
Lemma 5.2. Let $f:[0, T] \times \Omega \times \mathbb{R} \times \mathcal{P}_{2}\left(\mathbb{R}^{1+d}\right) \times \mathbb{R} \times \mathbb{R}^{d} \rightarrow \mathbb{R}$ be a continuous function in $(\eta, \mu, y, z)$ and satistying (H2), Then the sequence of functions

$$
f_{n}(s, \omega, \eta, \mu, y, z):=e s s \inf _{(\zeta, v, r, b) \in \mathbb{R} \times \mathcal{P}_{2}\left(\mathbb{R}^{1+d}\right) \times \mathbb{R} \times \mathbb{R}^{d}}\left\{f(s, \omega, \zeta, v, r, b)+n W_{2,+}(\mu, \nu)+n|\eta-\zeta|+n|y-r|+n|z-b|\right\}
$$

is well defined for $n \geqslant K$ and has the following properties

(i) Linear growth: for all $(s, \omega, \eta, \mu, y, z) \in[0, T] \times \Omega \times \mathbb{R} \times \mathcal{P}_{2}\left(\mathbb{R}^{1+d}\right) \times \mathbb{R} \times \mathbb{R}^{d},\left|f_{n}(s, \omega, \eta, \mu, y, z)\right| \leqslant C\left(1+W_{2}\left(\mu, \delta_{0}\right)+\right.$ $|\eta|+|y|+|z|)$

(ii) Monotonicity in $\mu$ : $f_{n}\left(s, \omega, \eta, \mu_{2}, y, z\right) \leqslant f_{n}\left(s, \omega, \eta, \mu_{1}, y, z\right)$, for $\mu_{1}, \mu_{2} \in \mathcal{P}_{2}\left(\mathbb{R}^{1+d}\right)$ with $F_{\mu_{2}} \geqslant F_{\mu_{1}}$, for all $(s, \omega, \eta, y, z) \in$ $[0, T] \times \Omega \times \mathbb{R} \times \mathbb{R} \times \mathbb{R}^{d}, n \geqslant 1$;

(iii) Monotonicity in $n$ : for any $(s, \omega, \eta, \mu, y, z) \in[0, T] \times \Omega \times \mathbb{R} \times \mathcal{P}_{2}\left(\mathbb{R}^{1+d}\right) \times \mathbb{R} \times \mathbb{R}^{d}, n \leqslant m, f_{n}(s, \omega, \eta, \mu, y, z) \leqslant$ $f_{m}(s, \omega, \eta, \mu, y, z)$;

(iv) Lipschtiz condition: for any $(s, \omega, \eta, \mu, y, z) \in[0, T] \times \Omega \times \mathbb{R} \times \mathcal{P}_{2}\left(\mathbb{R}^{1+d}\right) \times \mathbb{R} \times \mathbb{R}^{d},\left|f_{n}(s, \omega, \eta, \mu, y, z)-f_{n}\left(s, \omega, \eta_{1}, \mu_{1}, y_{1}, z_{1}\right)\right| \leqslant$ $n\left(W_{2}\left(\mu, \mu_{1}\right)+\left|\eta-\eta_{1}\right|+\left|y-y_{1}\right|+\left|z-z_{1}\right|\right)$;

(v) Strong convergence: If $\left(\eta_{n}, \mu_{n}, y_{n}, z_{n}\right) \rightarrow(\eta, \mu, y, z)$ in $\mathbb{R} \times \mathcal{P}_{2}\left(\mathbb{R}^{1+d}\right) \times \mathbb{R} \times \mathbb{R}^{d}$ as $n \rightarrow \infty$, then $f_{n}\left(s, \omega, \eta_{n}, \mu_{n}, y_{n}, z_{n}\right) \rightarrow$ $f(s, \omega, \eta, \mu, y, z)$ as $n \rightarrow \infty$.

From Lemma 5.2, for fixed $s$, we consider the sequence $f_{n}(s, \omega, \eta, \mu, y, z)$, and $g_{n}(s, \omega, v, \mu, y) n \geqslant 1$, related to $f$ and $g$, respectively. Also consider $h(s, \omega, \eta, \mu, y, z)=K\left(1+W_{2}\left(\mu, \delta_{0}\right)+|\eta|+|y|+|z|\right)$. It is obvious now that $f_{n}$ and $h$ are F-progressively measurable functions which are Lipschitz in $(\mu, y, z)$, uniformly in $(s, \omega)$. For $\xi \in L^{2}\left(\Omega, \mathcal{F}_{T} ; \mathbb{R}\right)$ we know from Proposition 4.5, for $n \geqslant K$, that the following mean-field BSDEs have a unique adapted solution

$$
\begin{gathered}
Y_{t}^{n}=\xi+\int_{t}^{T} f_{n}\left(s, \eta_{s}, P_{Y_{s}^{n}}, Y_{s}^{n}, Z_{s}^{n}\right) d s+\int_{t}^{T} g_{n}\left(s, \eta_{s}, P_{Y_{s}^{n}}, Y_{s}^{n}\right) d \Lambda_{s}-\int_{t}^{T} Z_{s}^{n} d B_{s}^{H}, \quad 0 \leqslant t \leqslant T . \\
U_{t}=|\xi|+\int_{t}^{T} h\left(s, \eta_{s}, P_{U_{s}}, U_{s}, V_{s}\right) d s+\int_{t}^{T} q\left(s, \eta_{s}, P_{U_{s}}, U_{s}\right) d \Lambda_{s}-\int_{t}^{T} V_{s} d B_{s}^{H}, \quad 0 \leqslant t \leqslant T .
\end{gathered}
$$

From Lemma 5.2, we know that $\left(f_{n}, g_{n}\right)$ and $(h, q)$ satisfy the assumptions of Proposition 4.5, therefore we have

$$
-U_{s} \leqslant Y_{s}^{m} \leqslant Y_{s}^{n} \leqslant U_{s}, P-\text { a.s, } s \in[0, T], \text { for all } n \geqslant m \geqslant K .
$$

The following two Lemmas have been implied in Proposition 4.5.

Lemma 5.3. There exists a conatant $C$ which depends on $K, T$ and $E\left[e^{\beta \Lambda_{T}} \xi^{2}\right]$, such that

$$
\begin{aligned}
& E\left(e^{\beta \Lambda_{s}}\left|Y_{t}^{n}\right|^{2}+\int_{t}^{T} e^{\beta \Lambda_{s}} s^{2 H-1}\left|Z_{s}^{n}\right|^{2} d s+\int_{t}^{T} e^{\beta \Lambda_{s}}\left|Y_{s}^{n}\right|^{2} d \Lambda_{s}\right) \leqslant C, \\
& E\left(e^{\beta \Lambda_{s}}\left|U_{t}\right|^{2}+\int_{t}^{T} e^{\beta \Lambda_{s}} s^{2 H-1}\left|V_{s}\right|^{2} d s+\int_{t}^{T} e^{\beta \Lambda_{s}}\left|U_{s}\right|^{2} d \Lambda_{s}\right) \leqslant C .
\end{aligned}
$$

Lemma 5.4. $\left(Y^{n}, Z^{n}\right), n \geqslant 1$, converges in $\mathcal{V}_{[0, T]}^{1 / 2} \times \mathcal{V}_{[0, T]}^{H}$.

Now, we give the main result of this paper:

Theorem 5.5. Let $\xi \in L^{2}\left(\Omega, \mathcal{F}_{T} ; \mathbb{R}\right)$. Assume (H2) holds. Then equation

$$
Y_{t}=\xi+\int_{t}^{T} f\left(s, \eta_{s}, P_{Y_{s}}, Y_{s}, Z_{s}\right) d s+\int_{t}^{T} g\left(s, \eta_{s}, P_{Y_{s}}, Y_{s}\right) d \Lambda_{s}-\int_{t}^{T} Z_{s}^{n} d B_{s}^{H}, \quad 0 \leqslant t \leqslant T .
$$

has an adapted solution $(Y, Z)$. Also, there is a minimal solution $\left(Y^{*}, Z^{*}\right)$ of $(9)$, in the sense that for any other solution $(Y, Z)$ of $(9)$, we have $Y_{s}^{*} \leqslant Y_{s}, s \in[0, T], P$-a.s. Moreover, for all $t \in[0, T]$,

$$
E\left(e^{\beta \Lambda_{s}}\left|Y_{t}\right|^{2}+\int_{t}^{T} e^{\beta \Lambda_{s}} s^{2 H-1}\left|Z_{s}\right|^{2} d s+\int_{t}^{T} e^{\beta \Lambda_{s}}\left|Y_{s}\right|^{2} d \Lambda_{s}\right) \leqslant C \Theta(t, T),
$$

where

$$
\Theta(t, T):=E\left(e^{\beta \Lambda_{T}}|\xi|^{2}+2 \int_{t}^{T} e^{\beta \Lambda_{s}}\left(1+E\left[\left(Y_{s}, Z_{s}\right)^{2}\right]\right) d s+\int_{t}^{T} e^{\beta \Lambda_{s}}\left|\eta_{s}\right|^{2} d s+2 \int_{t}^{T} e^{\beta \Lambda_{s}}\left(1+E\left[\left(Y_{s}\right)^{2}\right]\right) d \Lambda_{s}\right) .
$$


Proof. From (8) we have $Y^{n_{0}} \leqslant Y^{n} \leqslant U$ for all $n \geqslant n_{0} \geqslant K$. Moreover, $Y^{n} \rightarrow Y$ converges in $\mathcal{V}_{[0, T]}^{1 / 2}$, On the other hand, also $Z^{n} \rightarrow Z$ in $\mathcal{V}_{[0, T]}^{H}$.

Hence, thanks to (i) and (v) in Lemma 5.2, we get

$$
\begin{gathered}
f_{n}\left(s, \eta_{s}, P_{Y_{s}^{n}}, Y_{s}^{n}, Z_{s}^{n}\right) \rightarrow f\left(s, \eta_{s}, P_{Y_{s}}, Y_{s}, Z_{s}\right), \quad n \rightarrow \infty, \\
g_{n}\left(s, \eta_{s}, P_{Y_{s}^{n}}, Y_{s}^{n}\right) \rightarrow g\left(s, \eta_{s}, P_{Y_{s}}, Y_{s}\right) . \quad n \rightarrow \infty .
\end{gathered}
$$

Thus

$$
E\left(\int_{t}^{T} e^{\beta \Lambda_{s}}\left|f_{n}\left(s, \eta_{s}, P_{Y_{s}^{n}}, Y_{s}^{n}, Z_{s}^{n}\right)-f\left(s, \eta_{s}, P_{Y_{s}}, Y_{s}, Z_{s}\right)\right|^{2} d s\right) \rightarrow 0, \text { as } n \rightarrow \infty
$$

and

$$
E\left(\int_{t}^{T} e^{\beta \Lambda_{s}}\left(g_{n}\left(s, \eta_{s}, P_{Y_{s}^{n}}, Y_{s}^{n}\right)-g\left(s, \eta_{s}, P_{Y_{s}}, Y_{s}\right)\right) d \Lambda_{s}\right)^{2} \rightarrow 0, \text { as } n \rightarrow \infty .
$$

From Theorem 2.1, Lemma 4.5 and remark 3.2, we can get

$$
\begin{aligned}
& E\left(\int_{t}^{T} e^{\beta \Lambda_{s}}\left(Z_{s}^{n}-Z_{s}\right) d B_{s}^{H}\right)^{2}=E\left(\int_{t}^{T} e^{2 \beta \Lambda_{s}}\left(Z_{s}^{n}-Z_{s}\right)^{2} d s+\int_{t}^{T} \int_{t}^{T} \mathbb{D}_{r}^{H}\left(Z_{s}^{n}-Z_{s}\right) \mathbb{D}_{s}^{H}\left(Z_{r}^{n}-Z_{r}\right) d r d s\right) \\
= & E\left(\int_{t}^{T} e^{2 \beta \Lambda_{s}}\left(Z_{s}^{n}-Z_{s}\right)^{2} d s+2 \int_{t}^{T} \int_{s}^{T} \mathbb{D}_{r}^{H}\left(Z_{s}^{n}-Z_{s}\right) \mathbb{D}_{s}^{H}\left(Z_{r}^{n}-Z_{r}\right) d r d s\right) \\
\leqslant & E\left(\int_{t}^{T} e^{2 \beta \Lambda_{s}}\left(Z_{s}^{n}-Z_{s}\right)^{2} d s+2 M^{2} \int_{t}^{T} \int_{s}^{T}(s r)^{2 H-1}\left(Z_{s}^{n}-Z_{s}\right)\left(Z_{r}^{n}-Z_{r}\right) d r d s\right), \text { as } n \rightarrow \infty .
\end{aligned}
$$

On the other hand, from the BSDE (6) we can prove similarly that $E\left[\int_{0}^{T}\left|Y_{t}^{n}-Y_{t}^{m}\right| d t\right] \rightarrow 0$ as $n, m \rightarrow \infty$. Therefore, $Y$ has a continuous version, i.e. $Y \in \mathcal{V}_{[0, T]}^{1 / 2}$ and $E\left[\int_{0}^{T}\left|Y_{t}^{n}-Y_{t}\right| d t\right] \rightarrow 0$ as $n \rightarrow \infty$. Thus, taking the limit in (6), we get that $(Y, Z)$ solves (9).

Let $(\widehat{Y}, \widehat{Z}) \in \mathcal{V}_{[0, T]}^{1 / 2} \times \mathcal{V}_{[0, T]}^{H}$ be any solution of (9). From the comparison theorem we get that $Y_{s}^{n} \leqslant \widehat{Y}_{s}, s \in[0, T], P-$ a.s. for all $n \geqslant 1$, and therefore $Y_{s} \leqslant \widehat{Y}_{s}, s \in[0, T]$, P-a.s., that is, $Y$ is the minimal solution of (9).

\section{Acknowledgments}

This project was sponsored by National Natural Science Foundation of China (11901257) and the Fundamental Research Funds for the Jiangxi Normal University.

\section{References}

Bender, C. (2005). Exiplicit solutions of a calss linear fractional BSDEs. Syst. Control Lett., 54, 671-680.

Borkowska, K. J. (2013). Generalized BSDEs driven by fractional Brownian motion. Stat.Prob.Lett., 83, 805-811.

Biagini, F., Hu, Y., Øksendal, B., \& Sulem, A. (2002). A stochastic maximal principle for processes driven by fractional Brownian motion. Stoch. Process. Appl., 100, 233-253.

Buckdahn, R., Djehiche, B., Li, J., \& Peng, S. (2009). Mean-field backward stochastic differential equations. A limit approach, Ann. Probab., 37(4), 1524-1565.

Buckdahn, R., Li, J., \& Peng, S. (2009). Mean-field backward stochastic differential equations and related partial differential equations. Stochastic Process Appl., 119, 3133-3154.

Buckdahn, R., Li, J., Peng, S., \& Rainer, C. (2017). Mean-field stochastic differential equations and associated PDEs. Ann. Probab., 45(2), 824-878.

Decreusefond, L., \& Us̈tun̈el, A. S. (1998). Stoachstic analysis of the fractional Brownian motion. Potential Anal., 10, 177-214. 
Duncan, T. E., Hu, Y., \& Pasik-Duncan, B. (2000). Stochastic Calculus for fractional Brownian motion. I. Theory. SIAM. J. Control Option, 38, 582-621.

Du, H., Li., J., \& Wei, Q. (2011). Mean-field backward stochastic differential equations with continuous coefficients, in: Proceedings of the 30th Chinese Control Conference, Yantai, China, July 22-24.

Ferrante, M., \& Rovira, C. (2013).stochastic differential with non-negatively constraints driven by fractional Brownian motion. J. Evol. Equ., 13, 617-632.

Hu, Y. (2005). Integral transformations and anticipative calculus for fractional Brownian motion. Mem. Amer. Math. Soc., 825 , viii+127.

Hu, Y., \& Peng, S. (2009). Backward stochastic differential equations driven by fractional Brownian motion. SIAM J. Control Optim., 48, 1675-1700.

Hao., T., \& Li, J. (2016). Mean-field SDEs with jumps and nonlocal integral-PDEs. NoDEA Nonlinear Differential Equations Appl., 23(2), 1-51.

Juan, L., \& Min, H. (2016). Weak solutions of mean-field stochastic differential equations and application to zero-sum stochastic differential games. SIAM J. Control Optim., 54(3), 1826-1858.

Juan, L. (2018). Mean-field forward and backward SDEs with jumps and Associated nonlocal quasi-linear integral-PDEs. Stochastic Process. Appl., 128(9), 3118-3180.

Juan, L., Hao, L., \& Xiao, Z. (2018). General mean-field BSDEs with continuous coefficients. Journal of Mathematical Analysis and Applications, 466, 264-280.

Lions, P. L., \& Sznitman, A. S. (1984). Stochastic differential equations with reflecting boundary conditions. Comm. Pure Appl. Math., 37, 511-537.

Lepeltier, J. P., \& Martin, J. S. (1997). Backward stochastic differential equations with continuous coefficient. Statist. Probab. Lett., 32, 425-430.

Lasry, J. M., \& Lions, P. L. (2007). Mean field games. Jpn. J. Math, 2(1), 229-260.

Maticiuc, L., \& Nie, T. (2015). Fractional backward stochastic differential equations and Fractional backward variational inequalities. J. Theoret. Probab., 28, 337-395.

Ma, J., Protter, P., \& Yong, J.(1994). Solving forward-backward stochastic differential equations explicitly-a four step scheme. Probeb. Theo. Relat. Fields, 98, 339-359.

Nualart, D. (2006). Malliavin Calculus and Related Topics (2nd ed.). Springer-Verlag.

Pardoux, E., \& Peng, S. (1992). Backward SDE and quasilinear parabolic PDEs. In: Rozovskii, B. L., Sowers, R. Stochastic Partial Differential Equations and their Applications, LNCIS 176. Springer, New York, 200-217.

Pardoux, E., \& Zhang, S. (1998). Generalized BSDEs and non-Neumann boundary value problems. Probab. Theory Related. Fields, 110, 535-558.

Pipiras, V., \& Taqqu, M. S. (2000). Integration questions related to fractional Brownian motion. Probab. Theory Related Fields, 118, 251-291.

Pardoux, é., \& Peng, S. (1990). Adapted solution of a backward stochastic differential equation. Systems Control Letters, $14,55-61$.

Sow, A. B. (2014). BSDE with jumps and non Lipschitz coefficients: application to large deviations. Brazilian J. Probab. Stat., 8, 96-108.

Saisho, Y. (1987). Stochastic differential equations for multidimensional domains with refecting boundary, Probab. Theory Related Fields, 74, 455-477.

Young, L. C. (1936). An inquality of the Hölder type connected with Stietjies integration. Acta Math., 67, 251-282. 


\section{Copyrights}

Copyright for this article is retained by the author(s), with first publication rights granted to the journal.

This is an open-access article distributed under the terms and conditions of the Creative Commons Attribution license (http://creativecommons.org/licenses/by/4.0/). 